ELECTRONIC RESEARCH ANNOUNCEMENTS OF THE AMERICAN MATHEMATICAL SOCIETY

Volume 12, Pages 100-112 (August 10, 2006)

S $1079-6762(06) 00165-\mathrm{X}$

\title{
REPRESENTATIONS OF INTEGRAL QUADRATIC FORMS OVER DYADIC LOCAL FIELDS
}

\author{
CONSTANTIN N. BELI
}

(Communicated by Brian Conrey)

\begin{abstract}
In this paper we give necessary and sufficient conditions for the representations of quadratic lattices over arbitrary dyadic fields. Our result is given in terms of Bases of Norm Generators (BONGs, for short). However, they can be translated in terms of the more traditional Jordan decompositions.
\end{abstract}

\section{INTRODUCTION}

It has been known for a long time that the local theory of quadratic forms is considerably more difficult over dyadic fields, i.e., over finite extensions of $\mathbb{Q}_{2}$. Many problems that have simple answers and simple proofs over nondyadic local fields become significantly more complicated, both the statements and the proofs, when the base field is dyadic. The case of 2-adic fields (unramified extensions of $\mathbb{Q}_{2}$, i.e., local fields where 2 is a prime) is intermediate in the level of difficulty between the nondyadic and general dyadic case.

One reason for this phenomenon is the fact that the group of square classes $\dot{F} / \dot{F}^{2}$, which has only 4 elements when $F$ is nondyadic, has a more complicated structure in the dyadic case. Another reason is that the structure of the Jordan decomposition is more complicated in the dyadic case. One method to overcome this problem is the use of BONGs. The BONGs, short for "Bases of Norm Generators", were first introduced in $B$ as a way to describe lattices over dyadic fields. The BONGs, especially the good BONGs, behave to some extent like orthogonal bases. The usefulness of using BONGs rather than Jordan splittings was already seen in B], where we computed the spinor norm group $\theta\left(O^{+}(L)\right)$ for lattices $L$ over arbitrary dyadic fields. There is some strong evidence that by using BONGs, even though the proofs are still quite long (the result we announce here is a good example), the statements can be expressed in a very compact form, which is impossible to achieve if we use Jordan decompositions.

An important problem in the local theory of quadratic forms is the representation of local lattices. In 1958 O'Meara OM1 solved this problem in the nondyadic and the 2-adic cases. For the arbitrary dyadic case the same problem was solved in 1964 by Riehm $[\mathrm{R}$ ] under the assumption that the big lattice is modular. No progress towards a solution in the general dyadic case has been made since. In this paper we announce a complete solution of this problem. The answer is given in terms of good

Received by the editors January 23, 2006.

2000 Mathematics Subject Classification. Primary 11E08.

Key words and phrases. Integral quadratic forms, dyadic local fields.

(C)2006 American Mathematical Society Reverts to public domain 28 years from publication 
BONGs. However, it can be easily translated in terms of Jordan decompositions and the fundamental invariants of the two lattices as described in [OM, §9].

\section{BASES OF NORM GENERATORS (BONGs)}

In this section, we describe quadratic lattices in terms of good BONGs, which we first introduced in [B]. That paper, together with Chapter 9 of $[\mathrm{OM}]$, are prerequisites for this announcement. We now briefly recall the main notions and results from $[\mathrm{OM}]$ and $[\mathrm{B}]$ we use here. All quadratic spaces and lattices considered here will be over a dyadic local field $F$. We denote by $\mathcal{O}$ the ring of integers in $F$, $\mathfrak{p}$ the prime ideal, $\mathcal{O}^{\times}:=\mathcal{O} \backslash \mathfrak{p}$ the group of units, $e:=\operatorname{ord} 2$, and $\pi$ a fixed prime element. For $a \in \dot{F}$ we denote by $\mathcal{D}(a)$ its quadratic defect, $\mathcal{D}(a)=\bigcap_{x \in F}\left(a-x^{2}\right) \mathcal{O}$. If ord $a$ is odd, then $d(a)=a \mathcal{O}$. If $a \in \mathcal{O}^{\times}$, then $\mathcal{D}(a)$ is one of the ideals $0 \subset 4 \mathcal{O} \subset$ $4 \mathfrak{p}^{-1} \subset 4 \mathfrak{p}^{-3} \subset \ldots \subset \mathfrak{p}^{3} \subset \mathfrak{p}$.

For a given quadratic space $V$ we will denote by $B: V \times V \longrightarrow F$ the corresponding symmetric bilinear form and by $Q: V \longrightarrow F$ the quadratic form $Q(x)=B(x, x)$. All the quadratic forms here are assumed nondegenerate.

Let $L$ be a lattice of dimension $n$. We define $L^{\sharp}:=\{x \in F L \mid B(x, L) \subseteq \mathcal{O}\}$ and, for any fractionary ideal $\mathfrak{a}$, we define $L^{\mathfrak{a}}:=\{x \in L \mid B(x, L) \subseteq \mathfrak{a}\}=L \cap \mathfrak{a} L^{\sharp}$. If $L=L_{1} \perp L_{2}$, then $L^{\sharp}=L_{1}^{\sharp} \perp L_{2}^{\sharp}$ and $L^{\mathfrak{a}}=L_{1}^{\mathfrak{a}} \perp L_{2}^{\mathfrak{a}}$.

We introduce some fractionary ideals. The scale of $L$ is $\mathfrak{s} L:=B(L, L)$. The norm of $L$ is the ideal generated by $Q(L), \mathfrak{n} L:=Q(L) \mathcal{O}$. The volume of $L$ is the ideal generated by the determinant of $L, \operatorname{vol} L:=(\operatorname{det} L) \mathcal{O}$. We have $2 \mathfrak{s} L \subseteq \mathfrak{n} L \subseteq \mathfrak{s} L$ and $\operatorname{vol} L \subseteq(\mathfrak{s} L)^{n}$.

If $\mathfrak{s} L=\mathfrak{a}$ and $\operatorname{vol} L=\mathfrak{a}^{n}$ for some fractionary ideal $\mathfrak{a}$, we say that $L$ is $\mathfrak{a}$-modular. An equivalent condition is that $L^{\sharp}=\mathfrak{a}^{-1} L$. If $L=L_{1} \perp L_{2}$, then $L$ is a-modular iff both $L_{1}, L_{2}$ are $\mathfrak{a}$-modular.

An orthogonal splitting $L=L_{1} \perp \ldots \perp L_{t}$ is called a Jordan decomposition if all $L_{i}$ 's are modular and $\mathfrak{s} L_{1} \supset \ldots \supset \mathfrak{s} L_{t}$. Every lattice has Jordan decompositions. Moreover, $t, \operatorname{dim} L_{i}$ and $\mathfrak{s} L_{i}$ for $1 \leq i \leq t$ are independent of the choice of the Jordan decomposition.

If $x_{1}, \ldots, x_{n}$ is a sequence of mutually orthogonal vectors with $Q\left(x_{i}\right)=a_{i}$, we say that $V \cong\left[a_{1}, \ldots, a_{n}\right]$ relative to $x_{1}, \ldots, x_{n}$ if $V=F x_{1} \perp \ldots \perp F x_{n}$, and $L \cong\left\langle a_{1}, \ldots, a_{n}\right\rangle$ relative to $x_{1}, \ldots, x_{n}$ if $L=\mathcal{O} x_{1} \perp \ldots \perp \mathcal{O} x_{n}$.

We denote by $d: \dot{F} / \dot{F}^{2} \longrightarrow \mathbb{N} \cup\{\infty\}$ the order of the "relative quadratic defect" $d(a):=\operatorname{ord} a^{-1} \mathcal{D}(a)$. If $a=\pi^{R} \varepsilon$, then $d(a)=0$ if $R$ is odd, and $d(a)=d(\varepsilon)=$ ord $\mathcal{D}(\varepsilon)$ if $R$ is even. Thus $d(\dot{F})=\{0,1,3, \ldots, 2 e-1,2 e, \infty\}$. This function satisfies the domination principle $d(a b) \geq \min \{d(a), d(b)\}$, with equality if $d(a) \neq d(b)$.

Definition 2.1. An element $x$ of a lattice $L$ is called a norm generator of $L$ if $\mathfrak{n} L=Q(x) \mathcal{O}$. A sequence of vectors $x_{1}, \ldots, x_{n}$ is called a Basis of Norm Generators $(B O N G)$ for a lattice $L$ if $x_{1}$ is a norm generator for $L$ and $x_{2}, \ldots, x_{n}$ is a BONG for $p r_{x_{1}^{\perp}} L$.

It is known that a BONG uniquely determines a lattice [B, Corollary 2.6]. Therefore, if $x_{1}, \ldots, x_{n}$ is a BONG for $L$, we will write $L=\prec x_{1}, \ldots, x_{n} \succ$. If moreover $Q\left(x_{i}\right)=a_{i}$, we say that $L \cong \prec a_{1}, \ldots, a_{n} \succ$ relative to the BONG $x_{1}, \ldots, x_{n}$.

If $L$ is binary with $\mathfrak{n} L=\alpha \mathcal{O}$, we denote by $a(L):=\alpha^{-2} \operatorname{det} L$ and by $R(L):=$ ord vol $L-2 \operatorname{ord} \mathfrak{n} L=\operatorname{ord} a(L)$. Then $a(L) \in \dot{F} / \mathcal{O}^{\times 2}$ is an invariant of $L$ which determines the class of $L$ up to scaling. If $L \cong \prec \alpha, \beta \succ$, then $a(L)=\frac{\beta}{\alpha}$. The 
set of all possible values of $a(L)$, where $L$ is an arbitrary binary lattice is denoted by $\mathcal{A}=\mathcal{A}_{F}$. If $a \in \dot{F}$ and ord $a=R$, then $a \in \mathcal{A}$ if and only if $R+2 e \geq 0$ and $R+d(-a) \geq 0$.

Definition 2.2. A BONG $x_{1}, \ldots, x_{n}$ for a lattice $L$ is said to be a good BONG if ord $Q\left(x_{i}\right) \leq$ ord $Q\left(x_{i+2}\right)$ for all $1 \leq i \leq n-2$.

A sequence $x_{1}, \ldots, x_{n}$ of mutually orthogonal vectors with $Q\left(x_{i}\right)=a_{i}$ and ord $a_{i}=R_{i}$ is a good BONG for some lattice if and only if $R_{i} \leq R_{i+2}$ for all $1 \leq i \leq n-2$ and $a_{i+1} / a_{i} \in \mathcal{A}$ for all $1 \leq i \leq n-1$. By the above description of $\mathcal{A}$, the second condition is equivalent to $R_{i+1}-R_{i}+2 e \geq 0$ and $R_{i+1}-R_{i}+d\left(-a_{i} a_{i+1}\right) \geq 0$.

Definition 2.3. We say that $L=L_{1} \perp \ldots \perp L_{m}$ is a maximal norm splitting if each $L_{i}$ is either unary or binary modular, $\mathfrak{s} L_{1} \supseteq \ldots \supseteq \mathfrak{s} L_{m}$ and $\mathfrak{n} L_{i}=\mathfrak{n} L^{\mathfrak{s} L_{i}}$ for $1 \leq i \leq m$.

It follows from $[\mathrm{B}$, Lemma 4.3(iii)] that we can obtain a good BONG for $L$ by putting together the BONGs of the components of a maximal norm splitting of $L$. Conversely, every good BONG can be obtained in this way from some maximal norm splitting. By [B. Lemma 4.6], every lattice admits a maximal norm splitting and hence a good BONG. An algorithm to exhibit a maximal norm splitting of a lattice will be given in Section 7 .

\section{Classifichtion in terms of BONGs}

The problem of classifying lattices over arbitrary dyadic fields was solved by O'Meara in his renowned Theorem 93:28 in OM. Here we give an equivalent version of this theorem in term of BONGs. Our version has its own merit since its necessary and sufficient conditions are simpler than O'Meara's, and they require considerably less effort to verify. However, its main importance is that it is the first step towards solving the more complicated problem of representation.

First let us state O'Meara's theorem.

Given $\alpha, \beta \in \dot{F}$ and a fractionary ideal $\mathfrak{a}$ we say that $\alpha \cong \beta \bmod \mathfrak{a}$ if $\alpha / \beta \in \mathcal{O}^{\times}$ and $\alpha \equiv \beta \varepsilon^{2} \bmod \mathfrak{a}$ for some $\varepsilon \in \mathcal{O}^{\times}$.

If $L$ is a lattice, we define its norm group by $\mathfrak{g} L:=Q(L)+2 \mathfrak{s} L$. Thus, $\mathfrak{g} L$ is a subgroup of $(F,+)$. Let $\mathfrak{m} L$ be the largest fractionary ideal contained in $\mathfrak{g} L$. We define the weight of $L$ by $\mathfrak{w} L:=\mathfrak{p m} L+2 \mathfrak{s} L$. We have $\mathfrak{n} L \supseteq \mathfrak{g} L \supseteq \mathfrak{w} l \supseteq 2 \mathfrak{s} L$. An element of $\mathfrak{g} L$ of smallest order is called a norm generator of $L$. So $a$ is a norm generator for $L$ iff $a \in \mathfrak{g} L$ and $a \mathcal{O}=\mathfrak{n} L$. For any norm generator we have $\mathfrak{g} L=a \mathcal{O}^{2}+\mathfrak{w} L$. (Unfortunately there is some danger of confusion with the norm generators from the previous section. However, we can deduce which ones they are from the context. The norm generators from [OM] are scalars while those from $B$ are vectors.)

Now let $L=L_{1} \perp \ldots \perp L_{t}$ be a Jordan splitting. Denote $\mathfrak{s}_{i}=\mathfrak{s} L_{i}, \mathfrak{g}_{i}=$ $\mathfrak{g} L^{\mathfrak{s}_{i}}, \mathfrak{w}_{i}=\mathfrak{w} L^{\mathfrak{s}_{i}}$ and let $a_{i}$ be a norm generator for $L^{\mathfrak{s}_{i}}$. We have $\mathfrak{g}_{i}=a_{i} \mathcal{O}^{2}+$ $\mathfrak{w}_{i}$. Now $t, \operatorname{dim} L_{i}, \mathfrak{s}_{i}, \mathfrak{w}_{i}, a_{i}$ are called the fundamental invariants of $L$. If $L^{\prime}$ is a second lattice, let $t^{\prime}, \operatorname{dim} L_{i}^{\prime}, \mathfrak{s}_{i}^{\prime}, \mathfrak{w}_{i}^{\prime}, a_{i}^{\prime}$ be its fundamental invariants. Also let $\mathfrak{g}_{i}^{\prime}$ be the fundamental norm groups of $L^{\prime}$. Then we say that $L$ and $L^{\prime}$ have the same fundamental type if $t=t^{\prime}, \operatorname{dim} L_{i}=\operatorname{dim} L_{i}^{\prime}, \mathfrak{s}_{i}=\mathfrak{s}_{i}^{\prime}$ and $\mathfrak{g}_{i}=\mathfrak{g}_{i}^{\prime}$. Some equivalent conditions are $t=t^{\prime}, \operatorname{dim} L_{i}=\operatorname{dim} L_{i}^{\prime}, \mathfrak{s}_{i}=\mathfrak{s}_{i}^{\prime}, \mathfrak{w}_{i}=\mathfrak{w}_{i}^{\prime}$ and $a_{i} \cong a_{i}^{\prime} \bmod \mathfrak{w}_{i}$. 
Denote $s_{i}=\operatorname{ord} \mathfrak{s}_{i}$ and $u_{i}=\operatorname{ord} a_{i}$. We now introduce the ideals $\mathfrak{f}_{i}$ for $1 \leq i \leq$ $t-1$ by $\mathfrak{s}_{i}^{2} \mathfrak{f}_{i}=\sum \mathcal{D}(\alpha \beta)$ if $u_{i}+u_{i+1}$ is odd, and $\mathfrak{s}_{i}^{2} \mathfrak{f}_{i}=\sum \mathcal{D}(\alpha \beta)+2 \mathfrak{p}^{\left(u_{i}+u_{i+1}\right) / 2+\overline{s_{i}}}$ if $u_{i}+u_{i+1}$ is even. Here $\alpha$ and $\beta$ run over $\mathfrak{g}_{i}$ and $\mathfrak{g}_{i+1}$, respectively.

We now state O'Meara's theorem. Let $L$ be as above. If $L_{(i)}:=L_{1} \perp \ldots \perp L_{i}$, we call $L_{(1)} \subset \ldots \subset L_{(t)}$ the Jordan chain associated with the splitting $L=L_{1} \perp$ $\ldots \perp L_{t}$. Let $K$ be a second lattice and $K_{(1)} \subset \ldots \subset K_{\left(t^{\prime}\right)}$ a Jordan chain for $K$.

Theorem 3.1. We have $L \cong K$ iff $F L \cong F K, L$ and $K$ have the same fundamental type and the following conditions hold for $1 \leq i \leq t-1$ :

(i) $\operatorname{det} L_{(i)} / \operatorname{det} K_{(i)} \cong 1 \bmod \mathfrak{f}_{i}$.

(ii) $F L_{(i)} \rightarrow F K_{(i)} \perp\left[a_{i+1}\right]$ when $\mathfrak{f}_{i} \subset 4 a_{i+1} \mathfrak{w}_{i+1}^{-1}$.

(iii) $F L_{(i)} \rightarrow F K_{(i)} \perp\left[a_{i}\right]$ when $\mathfrak{f}_{i} \subset 4 a_{i} \mathfrak{w}_{i}^{-1}$.

We now translate O'Meara's theorem in terms of BONGs.

Suppose that $L \cong \prec a_{1}, \ldots, a_{n} \succ$ relative to a good BONG with ord $a_{i}=R_{i}$. For any $1 \leq i<n$, let $\alpha_{i}$ be the minimum of the following three sets of numbers:

$$
\begin{aligned}
& \left\{\left(R_{i+1}-R_{i}\right) / 2+e\right\}, \quad\left\{R_{i+1}-R_{j}+d\left(-a_{j} a_{j+1}\right): 1 \leq j \leq i\right\}, \\
& \left\{R_{j+1}-R_{i}+d\left(-a_{j} a_{j+1}\right): i \leq j<n\right\} .
\end{aligned}
$$

The numbers $R_{i}$ and $\alpha_{i}$ are invariants of the lattice $L$ since they do not depend on the choice of the good BONG. Therefore we denote them by $R_{i}(L)$ and $\alpha_{i}(L)$. The invariants $\alpha_{i}$ enjoy some properties such as:

(P1) The sequence $\left(R_{i}+\alpha_{i}\right)$ is increasing and $\left(-R_{i+1}+\alpha_{i}\right)$ is decreasing.

(P2) $\alpha_{i} \geq 0$ with equality if and only if $R_{i+1}-R_{i}=-2 e$.

(P3) If $R_{i+1}-R_{i} \leq 2 e$, then $\alpha_{i} \geq R_{i+1}-R_{i}$ with equality if and only if $R_{i+1}-$ $R_{i}=2 e$ or $R_{i+1}-R_{i}$ is odd.

(P4) $\alpha_{i}=\left(R_{i+1}-R_{i}\right) / 2+e$ if $R_{i+1}-R_{i} \geq 2 e$.

(P5) $\alpha_{i}$ is $<2 e,=2 e$ or $>2 e$ if $R_{i+1}-R_{i}$ is so.

(P6) If $R_{i-1}=R_{i+1}$, then $\alpha_{i-1}+\alpha_{i} \leq 2 e$.

(P7) $\alpha_{i}\left(L^{\sharp}\right)=\alpha_{n-i}(L)$.

The invariants $R_{i}$ and $\alpha_{i}$ can be written in terms of the fundamental invariants of $L$. Let $L=L_{1} \perp \ldots \perp L_{t}$ be a Jordan splitting, $L_{(1)} \subset \ldots \subset L_{(t)}$ the associated Jordan chain, and let $t, \operatorname{dim} L_{k}, \mathfrak{s}_{k}, \mathfrak{w}_{k}$ and $a_{k}$ be the fundamental invariants. (We denote the norm generators by $\mathrm{a}_{k}$, not $a_{k}$, in order to prevent confusion with the $a_{i}$ 's from $L \cong \prec a_{1}, \ldots, a_{n} \succ$.) Let $n_{k}=\operatorname{dim} L_{(k)}, r_{k}=\operatorname{ord} \mathfrak{s}_{k}, u_{k}=$ ord $\mathrm{a}_{k}$, and let $\mathfrak{f}_{k}$ be the invariants described above. Then for any $n_{k-1}+1 \leq i \leq n_{k}$, we have

$$
R_{i}= \begin{cases}u_{k} & \text { if } i \equiv n_{k-1}+1(\bmod 2) \\ 2 r_{k}-u_{k} & \text { if } i \equiv n_{k-1}(\bmod 2)\end{cases}
$$

Alternatively, $R_{i}=u_{k}$ if $i \equiv n_{k}+1(\bmod 2)$, and $R_{i}=2 r_{k}-u_{k}$ if $i \equiv n_{k}(\bmod 2)$.

If $n_{k-1}<i<n_{k}$, then $R_{i}+\alpha_{i}=$ ord $\mathfrak{w}_{k}$ and $-R_{i+1}+\alpha_{i}=\operatorname{ord} \mathfrak{w}_{t-k}^{\sharp}$. If $i=n_{k}$, then $\alpha_{i}=$ ord $\mathfrak{f}_{k}$ unless $R_{i+1}-R_{i}$ is odd and $>2 e$. In this case, $\alpha_{i}=$ $\left(R_{i+1}-R_{i}\right) / 2+e$ but ord $\mathfrak{f}_{k}=R_{i+1}-R_{i}$. In particular, $\alpha_{i}$ and ord $\mathfrak{f}_{k}$ are both $>2 e$, and hence both of the conditions $d(a) \geq \alpha_{i}$ and $d(a) \geq$ ord $\mathfrak{f}_{k}$ are equivalent to $a \in \dot{F}^{2}$. In the case when $L_{k}$ is unary we have $\mathrm{a}_{k}^{-1} \mathfrak{w}_{k}=\mathfrak{f}_{k-1}+\mathfrak{f}_{k}+2 \mathcal{O}$. If we denote $i=n_{k}=n_{k-1}+1$, we get $-R_{i}+$ ord $\mathfrak{w}_{k}=\min \left\{\right.$ ord $\mathfrak{f}_{k-1}$, ord $\left.\mathfrak{f}_{k}, e\right\}$, which implies ord $\mathfrak{w}_{k}=\min \left\{R_{i}+\alpha_{i-1}, R_{i}+\alpha_{i}, R_{i}+e\right\}$. (If $k=1$, so $i=1$, then we ignore $\mathfrak{f}_{k-1}$ and $\alpha_{i-1}$ above. If $k=t$, so $i=n$, then we ignore $\mathfrak{f}_{k}$ and $\alpha_{i}$.) 
In terms of BONGs, O'Meara's Theorem 93:28 can be stated as follows:

Theorem 3.2. Let $L \cong \prec a_{1}, \ldots, a_{n} \succ$ and $K \cong \prec b_{1}, \ldots, b_{n} \succ$ relative to some good BONGs with $R_{i}(L)=R_{i}, R_{i}(K)=S_{i}, \alpha_{i}(L)=\alpha_{i}$ and $\alpha_{i}(K)=\beta_{i}$. If $F L \cong F K$, then $L \cong K$ if and only if:

(i) $R_{i}=S_{i}$ for $1 \leq i \leq n$.

(ii) $\alpha_{i}=\beta_{i}$ for $1 \leq i \leq n-1$.

(iii) $d\left(a_{1} \cdots a_{i} b_{1} \cdots b_{i}\right) \geq \alpha_{i}$ for $1 \leq i \leq n-1$.

(iv) $\left[b_{1}, \ldots, b_{i-1}\right] \rightarrow\left[a_{1}, \ldots, a_{i}\right]$ for any $2 \leq i \leq n-1$ such that $\alpha_{i-1}+\alpha_{i}>2 e$.

The four conditions are equivalent to the conditions of O'Meara's classification theorem [OM, 93:28]. Namely, if $K=K_{1} \perp \ldots \perp K_{t^{\prime}}$ is a Jordan splitting, and $\mathfrak{s}_{k}^{\prime}, \mathfrak{w}_{k}^{\prime}, \mathrm{b}_{k}$ and $\mathfrak{f}_{k}^{\prime}$ are the invariants for $K$, then: (i) is equivalent to $t=t^{\prime}, \operatorname{dim} L_{k}=$ $\operatorname{dim} K_{k}, \mathfrak{s}_{k}=\mathfrak{s}_{k}^{\prime}$ and $\mathrm{a}_{k} \mathcal{O}=\mathrm{b}_{k} \mathcal{O}$; assuming that (i) holds, (ii) is equivalent to $\mathfrak{w}_{k}=$ $\mathfrak{w}_{k}^{\prime}$ and $\mathfrak{f}_{k}=\mathfrak{f}_{k}^{\prime}$; assuming that (i) and (ii) hold, (iii) is equivalent to $\mathrm{a}_{k} \cong \mathrm{b}_{k} \bmod \mathfrak{w}_{k}$ and condition (i) of [OM, 93:28]; assuming that (i)-(iii) hold, (iv) is equivalent to conditions (ii) and (iii) of [OM, 93:28]. More details of the proof of Theorem 3.2 will be presented in B1].

\section{Representations in terms of BONGs}

4.1. The invariants $d\left[\varepsilon a_{1, i} b_{1, j}\right]$ and $d\left[\varepsilon a_{i, j}\right]$. If $x_{1}, x_{2}, \ldots$ is a sequence in $\dot{F}$ and $j \geq i$, we define $x_{i, j}:=x_{i} \cdots x_{j}$. By convention, we set $x_{i, i-1}:=1$.

Definition 4.1. Suppose that $M \cong \prec a_{1}, \ldots, a_{m} \succ$ and $N \cong \prec b_{1}, \ldots, b_{n} \succ$ relative to some good BONGs. Let $\alpha_{i}(M)=\alpha_{i}$ and $\alpha_{i}(N)=\beta_{i}$. Then for any $0 \leq i \leq m, 0 \leq j \leq n$ and $\varepsilon \in \dot{F}$, we define

$$
d\left[\varepsilon a_{1, i} b_{1, j}\right]:=\min \left\{d\left(\varepsilon a_{1, i} b_{1, j}\right), \alpha_{i}, \beta_{j}\right\} .
$$

(If $i=0$ or $m$, we ignore $\alpha_{i}$; if $j=0$ or $n$, we ignore $\beta_{j}$.)

For $0 \leq i-1 \leq j \leq m$, we define

$$
d\left[\varepsilon a_{i, j}\right]:=d\left[\varepsilon a_{1, j} a_{1, i-1}\right]=\min \left\{d\left(\varepsilon a_{i, j}\right), \alpha_{i-1}, \alpha_{j}\right\} .
$$

(If $i=1$ or $m+1$, we ignore $\alpha_{i-1}$; if $j=0$ or $m$, we ignore $\alpha_{j}$.)

Lemma 4.2. $d\left[\varepsilon a_{1, i} b_{1, j}\right]$ and $d\left[\varepsilon a_{i, j}\right]$ are independent of the choice of the BONGs.

The proof of this lemma uses Theorem 3.2 (ii). Therefore, if $M \cong \prec a_{1}^{\prime}, \ldots, a_{m}^{\prime} \succ$ and $N \cong \prec b_{1}^{\prime}, \ldots, b_{n}^{\prime} \succ$ relative to some other good BONGs, then $d\left(a_{1, i} a_{1, i}^{\prime}\right) \geq \alpha_{i}$ and $d\left(b_{1, j} b_{1, j}^{\prime}\right) \geq \beta_{j}$.

The invariants $d\left[\varepsilon a_{1, i} b_{1, j}\right]$ satisfy the following domination principle: If we have a third lattice $K \cong \prec c_{1}, \ldots, c_{k} \succ$ with $\alpha_{i}(K)=\gamma_{i}$, then

$$
d\left[\varepsilon \varepsilon^{\prime} a_{1, i} c_{1, \ell}\right] \geq \min \left\{d\left[\varepsilon a_{1, i} b_{1, j}\right], d\left[\varepsilon^{\prime} b_{1, j} c_{1, \ell}\right]\right\} .
$$

Similarly, if some of the lattices $M, N, K$ are the same, we get inequalities involving also terms of the form $d\left[\varepsilon a_{i, j}\right]$. For example, since $d\left[\varepsilon^{\prime} a_{1, i} a_{1, \ell}\right]=d\left[\varepsilon^{\prime} a_{i+1, \ell}\right]$, the numbers $d\left[\varepsilon a_{1, i} b_{1, j}\right], d\left[\varepsilon^{\prime} a_{i+1, \ell}\right]$ and $d\left[\varepsilon \varepsilon^{\prime} a_{1, \ell} b_{1, j}\right]$ satisfy the domination principle. 
4.2. Statement of the main theorem. It is assumed throughout this subsection that $M \cong \prec a_{1}, \ldots, a_{m} \succ$ and $N \cong \prec b_{1}, \ldots, b_{n} \succ$ relative to some good BONGs. Let $R_{i}=R_{i}(M)=\operatorname{ord} a_{i}, S_{i}=R_{i}(N)=\operatorname{ord} b_{i}, \alpha_{i}=\alpha_{i}(M)$ and $\beta_{i}=\alpha_{i}(N)$.

Definition 4.3. For any $1 \leq i \leq \min \{m-1, n\}$, we define

$$
\begin{aligned}
& A_{i}:=\min \left\{\left(R_{i+1}-S_{i}\right) / 2+e, R_{i+1}-S_{i}+d\left[-a_{1, i+1} b_{1, i-1}\right]\right. \\
&\left.R_{i+1}+R_{i+2}-S_{i-1}-S_{i}+d\left[a_{1, i+2} b_{1, i-2}\right]\right\} .
\end{aligned}
$$

If $n \leq m-2$, we define

$$
\begin{aligned}
& S_{n+1}+A_{n+1}:=\min \left\{R_{n+2}+d\left[-a_{1, n+2} b_{1, n}\right],\right. \\
&\left.R_{n+2}+R_{n+3}-S_{n}+d\left[a_{1, n+3} b_{1, n-1}\right]\right\} .
\end{aligned}
$$

In the above definition, we ignore those terms that do not make sense. That is, if $i=1$ or $m-1$, then we ignore $R_{i+1}+R_{i+2}-S_{i-1}-S_{i}+d\left[a_{1, i+2} b_{1, i-2}\right]$ from the definition of $A_{i}$; and if $n=m-2$, then we ignore $R_{n+2}+R_{n+3}-S_{n}+d\left[a_{1, n+3} b_{1, n-1}\right]$ from the definition of $S_{n+1}+A_{n+1}$.

In view of Lemma 4.2, $A_{i}$ does not depend on the BONGs. Hence we can write $A_{i}=A_{i}(M, N)$. The same holds for $S_{n+1}+A_{n+1}$.

Remark 4.4. The definition of $S_{n+1}+A_{n+1}$ is justified as follows. Since $S_{n+1}$ is not defined, we assume that $S_{n+1} \gg 0$ is arbitrary. If we put $i=n+1$ in the formula for $A_{i}$ and add $S_{n+1}$, we get $S_{n+1}+A_{n+1}=\min \left\{\left(R_{n+2}+S_{n+1}\right) / 2+e, R_{n+2}+\right.$ $\left.d\left[-a_{1, n+2} b_{1, n}\right], R_{n+2}+R_{n+3}-S_{n}+d\left[a_{1, n+3} b_{1, n-1}\right]\right\}$. But $\left(R_{n+2}+S_{n+1}\right) / 2+e \longrightarrow \infty$ as $S_{n+1} \longrightarrow \infty$; so it can be removed.

The following theorem, formulated in terms of invariants derived from good BONGs, is our solution to the representation problem of quadratic lattices over arbitrary dyadic local fields. A complete proof will be presented in B2].

Theorem 4.5. Assume that $F N \rightarrow F M$. Then $N \rightarrow M$ if and only if:

(i) For any $1 \leq i \leq n$, we have either $R_{i} \leq S_{i}$ or $1<i<m$ and $R_{i}+R_{i+1} \leq$ $S_{i-1}+S_{i}$.

(ii) For any $1 \leq i \leq \min \{m-1, n\}$ we have $d\left[a_{1, i} b_{1, i}\right] \geq A_{i}$.

(iii) For any $1<i \leq \min \{m-1, n+1\}$ such that $R_{i+1}>S_{i-1}$ and $A_{i-1}+A_{i}>$ $2 e+R_{i}-S_{i}$, we have $\left[b_{1}, \ldots, b_{i-1}\right] \rightarrow\left[a_{1}, \ldots, a_{i}\right]$.

(iv) For any $1<i \leq \min \{m-2, n+1\}$ such that $S_{i} \geq R_{i+2}>S_{i-1}+2 e \geq$ $R_{i+1}+2 e$, we have $\left[b_{1}, \ldots, b_{i-1}\right] \rightarrow\left[a_{1}, \ldots, a_{i+1}\right]$. (If $i=n+1$, we ignore the condition $S_{i} \geq R_{i+2}$.)

Note that if $n \leq m-2$ and $i=n+1$, then the inequality $A_{i-1}+A_{i}>2 e+R_{i}-S_{i}$ from Theorem 4.5 (iii) can be written as $A_{n}+S_{n+1}+A_{n+1}>2 e+R_{n+1}$. It makes sense since $S_{n+1}+A_{n+1}$ is defined although $S_{n+1}$ and $A_{n+1}$ are not.

4.3. Some remarks. Conditions (i)-(iv) of Theorem 4.5 have some consequences.

The $A_{i}$ 's are generalizations of the $\alpha_{i}$ invariants. One can prove that $A_{i}(L, L)=$ $\alpha_{i}(L)$. If, in addition, $m=n$, then we have a property similar to (P7): $A_{i}(M, N)=$ $A_{n-i}\left(N^{\sharp}, M^{\sharp}\right)$.

When $m=n$, conditions (i) and (iii) of Theorem 4.5 for the lattices $N^{\sharp}, M^{\sharp}$ at an index $i$ are equivalent to the similar conditions for $M, N$ at the index $n+1-i$, while conditions (ii) and (iv) for $N^{\sharp}, M^{\sharp}$ at $i$ are equivalent to those for $M, N$ at $n-i$. 
Lemma 4.6. If $M, N$ satisfy condition (i) of Theorem 4.5, then:

(i) $R_{i}+R_{i+1} \leq S_{i}+S_{i+1}$ for $1 \leq i \leq n-1$.

(ii) If $R_{i}+R_{i+1}>S_{i-1}+S_{i}$, then $R_{i+1}>S_{i-1}$.

(iii) If $R_{i+1} \geq S_{i-1}$, then $R_{i} \leq S_{i}$.

In some cases conditions (ii) and (iii) of Theorem 4.5 need not be verified; see Lemmas 4.8 and 4.9 below.

Definition 4.7. An index $1 \leq i \leq \min \{m, n+1\}$ is called essential for the pair of lattices $M, N$ if $R_{i+1}>S_{i-1}$ and $R_{i+1}+R_{i+2}>S_{i-2}+S_{i-1}$. (The inequalities that do not make sense because $R_{i+1}, R_{i+2}, S_{i-2}$ or $S_{i-1}$ is not defined are ignored.)

Lemma 4.8. Condition (ii) of Theorem 4.1 is vacuous at an index $i$ if both $i$ and $i+1$ are not essential.

Lemma 4.9. Condition (iii) of Theorem 4.1 is vacuous at an index $i$ if $i$ is not essential.

\section{RePRESEntations in terms OF JoRdAN DECOMPOSITIONS}

Let $L \cong \prec a_{1}, \ldots, a_{n} \succ$ be relative to a good BONG, and let $L=L_{1} \perp \ldots \perp L_{t}$ be a Jordan decomposition. Let $L_{(k)}, \mathfrak{s}_{k}, a_{k}, \mathfrak{w}_{k}$ and $\mathfrak{f}_{k}$ be defined as in Section 3 , and let $R_{i}(L)=R_{i}$ and $\alpha_{i}(L)=\alpha_{i}$. Recall that for $j \geq i, a_{i, j}$ is defined to be the product $a_{i} \cdots a_{j}$. Also, $a_{i, i-1}$ is set to be equal to 1 . For the sake of convenience, the space $\left[a_{1}, \ldots, a_{i}\right]$ is interpreted as the 0 space when $i=0$.

Definition 5.1. For any $1 \leq i \leq n-1$, an element $X_{i} \in \dot{F} / \dot{F}^{2}$ is called an approximation for $a_{1, i}$, denoted $X_{i} \sim a_{1, i}$, if $d\left(a_{1, i} X_{i}\right) \geq \alpha_{i}$. For $i=0$ or $n$, we write $X_{0} \sim a_{1,0}$ if $X_{0}=1$, and $X_{n} \sim a_{1, n}$ if $X_{n}=\operatorname{det} F L$ (in $\left.\dot{F} / \dot{F}^{2}\right)$.

Definition 5.2. Let $1 \leq i \leq n-1$ and let $V_{i}$ be a quadratic space of dimension $i$ such that $\operatorname{det} V_{i}$ is an approximation for $a_{1, i}$. We say that $V_{i}$ is an approximation to the left for $\left[a_{1}, \ldots, a_{i}\right]$ if $\alpha_{i-1}+\alpha_{i}>2 e$ or $i=1$ implies $\left[a_{1}, \ldots, a_{i-1}\right] \rightarrow V_{i}$; an approximation to the right if $\alpha_{i}+\alpha_{i+1}>2 e$ or $i=n-1$ implies $V_{i} \rightarrow\left[a_{1}, \ldots, a_{i+1}\right]$; an approximation if it is an approximation both to the left and right. We denote this by $V_{i} \sim_{l}\left(\right.$ resp. $\sim_{r}$ or $\left.\sim\right)\left[a_{1}, \ldots, a_{i}\right]$. By convention, if $i=0$ or $n$, we say that $V_{i} \sim\left[a_{1}, \ldots, a_{i}\right]$ if $V_{0} \cong 0$, resp. $V_{n} \cong F L$.

Note that Definitions 5.1 and 5.2 refer to the good BONG at hand. However, one can prove that these definitions are independent of the choice of the good BONG for $L$.

We want to obtain approximations for $a_{1, i}$ and $\left[a_{1}, \ldots, a_{i}\right]$ in terms of the Jordan decompositions.

Lemma 5.3. Let $n_{k-1} \leq i<n_{k}$ with $1 \leq k \leq t$.

(i) If $i \equiv n_{k-1}(\bmod 2)$, then $X_{i}=(-1)^{\left(i-n_{k-1}\right) / 2} \operatorname{det} F L_{(k-1)}$ approximates $a_{1, i}$.

(ii) If $i \equiv n_{k-1}+1(\bmod 2)$, then $X_{i}=\mathrm{a}_{k} \operatorname{det} F L_{(k-1)}$ approximates $a_{1, i}$. Alternatively, if $n_{k-1}<i \leq n_{k}$ with $1 \leq k \leq t$, then

(i') If $i \equiv n_{k}(\bmod 2)$, then $X_{i}=(-1)^{\left(n_{k}-i\right) / 2} \operatorname{det} F L_{(k)}$ approximates $a_{1, i}$.

(ii') If $i \equiv n_{k}+1(\bmod 2)$, then $X_{i}=\mathrm{a}_{k} \operatorname{det} F L_{(k)}$ approximates $a_{1, i}$. 
Before finding approximates for $\left[a_{1}, \ldots, a_{i}\right]$, note that if $R_{i-1}=R_{i+1}$, then, by (P6), $\alpha_{i-1}+\alpha_{i} \leq 2 e$. So for any $V_{i}$ with $\operatorname{dim} V_{i}=i$ and $\operatorname{det} V_{i} \sim a_{1, i}$, we have $V_{i} \sim_{l}\left[a_{1}, \ldots, a_{i}\right]$. Similarly if $R_{i}=R_{i+2}$, then $V_{i} \sim_{r}\left[a_{1}, \ldots, a_{i}\right]$ for any $V_{i}$ with $\operatorname{dim} V_{i}=i$ and $\operatorname{det} V_{i} \sim a_{1, i}$.

If $W \rightarrow V$ are two quadratic spaces, then we denote by $V \top W$ a quadratic space $U$ such that $V \cong W \perp U$.

Lemma 5.4. Let $0 \leq i \leq n$. Then:

(i) If $i=n_{k}$ and $V_{i}=F L_{(k)}$, then $V_{i} \sim\left[a_{1}, \ldots, a_{i}\right]$.

(ii) If $i=n_{k}+1<n_{k+1}$ and $V_{i}=F L_{(k)} \perp\left[\mathrm{a}_{k+1}\right]$, then $V_{i} \sim_{l}\left[a_{1}, \ldots, a_{i}\right]$. If moreover $R_{i}=R_{i+2}$, then $V_{i} \sim\left[a_{1}, \ldots, a_{i}\right]$.

(iii) If $i=n_{k}-1>n_{k-1}$ and $V_{i}=F L_{(k)} \top\left[\mathrm{a}_{k}\right]$, then $V_{i} \sim_{r}\left[a_{1}, \ldots, a_{i}\right]$. If moreover $R_{i-1}=R_{i+1}$, then $V_{i} \sim\left[a_{1}, \ldots, a_{i}\right]$.

Lemma 5.5. Suppose that $0 \leq i \leq n$ is not of the form $i=n_{k}$ and that neither $R_{i-1}=R_{i+1}$ nor $R_{i}=R_{i+2}$ holds.

(i) Then $i=n_{k-1}+1=n_{k}-1$ for some $k$ such that $L_{k}$ is binary, and we have $\mathfrak{n} L_{k}=\mathfrak{n} L^{\mathfrak{s}_{k}}$ regardless of the choice of the Jordan decomposition.

(ii) Assume that $\mathrm{a}_{k} \in Q\left(F L_{k}\right)$. (In particular this happens if we take $\mathrm{a}_{k} \in$ $Q\left(L_{k}\right)$ with $\mathfrak{n} L_{k}=\mathrm{a}_{k} \mathcal{O}$.) Then both $F L_{(k-1)} \perp\left[\mathrm{a}_{k}\right]$ and $F L_{(k)} \top\left[\mathrm{a}_{k}\right]$ approximate $\left[a_{1}, \ldots, a_{i}\right]$.

In all the other cases not described in Lemmas 5.4 and 5.5, we have both $R_{i-1}=$ $R_{i+1}$ and $R_{i}=R_{i+2}$. So $V_{i} \cong\left[a_{1}, \ldots, a_{i}\right]$ whenever $\operatorname{dim} V_{i}=i$ and $\operatorname{det} V_{i} \sim a_{1, i}$.

Suppose now that we have two lattices $M, N$. Let $X_{i}, Y_{i}, V_{i}$ and $W_{i}$ be approximations for $a_{1, i}, b_{1, i},\left[a_{1}, \ldots, a_{i}\right]$ and $\left[b_{1}, \ldots, b_{i}\right]$, respectively. Then in Definition 4.1, we can replace the products $a_{1, i}$ and $b_{1, i}$ by their approximations. So $d\left[\varepsilon a_{1, i} b_{1, j}\right]=$ $d\left[\varepsilon X_{i} Y_{j}\right]:=\min \left\{d\left(\varepsilon X_{i} Y_{j}\right), \alpha_{i}, \beta_{j}\right\}$. Similarly, since $d\left[\varepsilon a_{i, j}\right]=d\left[\varepsilon a_{1, i-1} a_{1, j}\right]$, we have $d\left[\varepsilon a_{i, j}\right]=d\left[\varepsilon X_{i-1} X_{j}\right]:=\min \left\{d\left(\varepsilon X_{i-1} X_{j}\right), \alpha_{i-1}, \alpha_{j}\right\}$. In particular, in the definition of $A_{i}$ (Definition 4.3), we can replace $d\left[-a_{1, i+1} b_{1, i-1}\right]$ and $d\left[a_{1, i+2} b_{1, i-2}\right]$ by $d\left[-X_{i+1} Y_{i-1}\right]$ and $d\left[X_{i+2} Y_{i-2}\right]$, respectively. So $A_{i}$ can be calculated by using approximations. The same holds for $S_{n+1}+A_{n+1}$.

Lemma 5.6. Assume that $M$ and $N$ satisfy conditions (i) and (ii) of Theorem 4.5. Let $1<i \leq \min \{m-1, n+1\}$ such that $R_{i+1}>S_{i-1}$ and $A_{i-1}+A_{i}>2 e+R_{i}-S_{i}$. Then $\left[b_{1}, \ldots, b_{i-1}\right] \rightarrow\left[a_{1}, \ldots, a_{i}\right]$ if and only if $W_{i-1} \rightarrow V_{i}$.

Lemma 5.7. If $R_{i+1}>R_{i}+2 e$ or $i \in\{0, m\}$, then $V_{i} \sim\left[a_{1}, \ldots, a_{i}\right]$ implies $V_{i} \cong\left[a_{1}, \ldots, a_{i}\right]$. Similarly, if $S_{i+1}>S_{i}+2 e$ or $i \in\{0, n\}$, then $W_{i} \sim\left[b_{1}, \ldots, b_{i}\right]$ implies $W_{i} \cong\left[b_{1}, \ldots, b_{i}\right]$.

As a consequence, if there is an $i$ such that $1<i \leq \min \{m-2, n+1\}$ and $S_{i} \geq R_{i+2}>S_{i-1}+2 e \geq R_{i+1}+2 e$, then we have both $W_{i-1} \cong\left[b_{1}, \ldots, b_{i-1}\right]$ and $V_{i+1} \cong\left[a_{1}, \ldots, a_{i+1}\right]$. (Recall that if $i=n+1$, we ignore the condition $S_{i} \geq R_{i+2}$.) It is because $S_{i}>S_{i-1}+2 e\left(\right.$ or $i-1=n$ ) and $R_{i+2}>R_{i+1}+2 e$. Thus $\left[b_{1}, \ldots, b_{i-1}\right] \rightarrow\left[a_{1}, \ldots, a_{i+1}\right]$ if and only if $W_{i-1} \rightarrow V_{i+1}$. Consequently,

Proposition 5.8. Assume that $M$ and $N$ satisfy condition (i) of Theorem 4.5. Then conditions (ii), (iii) and (iv) of Theorem 4.5 are equivalent to:

(ii') For any $1 \leq i \leq \min \{m-1, n\}$, we have $d\left[X_{i} Y_{i}\right] \geq A_{i}$.

(iii') For any $1<i \leq \min \{m-1, n+1\}$ such that $R_{i+1}>S_{i-1}$ and $A_{i-1}+A_{i}>$ $2 e+R_{i}-S_{i}$, we have $W_{i-1} \rightarrow V_{i}$. 
(iv') For any $1<i \leq \min \{m-2, n+1\}$ such that $S_{i} \geq R_{i+2}>S_{i-1}+2 e \geq$ $R_{i+1}+2 e$, we have $W_{i-1} \rightarrow V_{i+1}$.

Therefore, Theorem 4.5 can be stated in terms of the invariants $R_{i}, S_{i}, \alpha_{i}, \beta_{i}$ and the approximations $X_{i}, Y_{i}, V_{i}, W_{i}$. Since all these can be deduced from the Jordan decompositions, Theorem 4.5 can be stated in terms of Jordan decompositions. However, a detailed writing of all the cases that occur might be very lengthy.

\section{Outline of the PRoOF}

In this section, we outline the strategy of the proof of Theorem 4.5. The proof consists of several steps:

1. We first reduce to the case when $m=n$. To do this we assume that $F N \subset F M$ and take a lattice $K$ on $F N^{\perp}$ in $F M$. Note that $N \rightarrow M$ if and only if $N \perp \mathfrak{p}^{s} K \rightarrow M$ for $s \gg 0$. We then prove that $M$ and $N$ satisfy conditions (i)-(iv) of Theorem 4.5 if and only if $M$ and $N \perp \mathfrak{p}^{s} K$ do.

2. Prove that conditions (i)-(iv) of Theorem 4.5) are independent of the choice of the BONGs. To do so, let us assume that we have $M \cong \prec a_{1}^{\prime}, \ldots, a_{m}^{\prime} \succ$ and $N \cong \prec b_{1}^{\prime}, \ldots, b_{n}^{\prime} \succ$ relative to some other good BONGs. By Theorem [3.2(iii) and (iv), $a_{1, i}^{\prime}$ and $\left[a_{1}^{\prime}, \ldots, a_{i}^{\prime}\right]$ are approximations for $a_{1, i}$ and $\left[a_{1}, \ldots, a_{i}\right]$, respectively. The same holds for $b_{1, i}^{\prime}$ and $\left[b_{1}^{\prime}, \ldots, b_{i}^{\prime}\right]$. Then we use Proposition 5.8 with $X_{i}=a_{1, i}^{\prime}, Y_{i}=b_{1, i}^{\prime}, V_{i}=\left[a_{1}^{\prime}, \ldots, a_{i}^{\prime}\right]$ and $W_{i}=\left[b_{1}^{\prime}, \ldots, b_{i}^{\prime}\right]$.

This allows us to say that $N \leq M$ if $F N \rightarrow F M$ and $M, N$ satisfy conditions (i)-(iv) of Theorem 4.5. Our goal is to prove that

$$
\text { (*) } \quad N \rightarrow M \text { if and only } N \leq M \text {. }
$$

In view of step 1, we may assume that $F M=F N$.

The proof of necessity of $(*)$ consists of two steps:

N1. Prove that $\leq$ defined above is transitive for lattices on the same quadratic space.

N2. Prove that $M=N$ or $[M: N]=\mathfrak{p}$ implies $N \leq M$.

It is clear why these two steps are enough. For, if $N \subset M$, there is a sequence $M=M_{0} \supset \ldots \supset M_{k}=N$ with $\left[M_{k}: M_{k+1}\right]=\mathfrak{p}$. It follows from N2 that $M_{1} \leq M_{0}=M, M_{2} \leq M_{1}, \ldots, N=M_{k} \leq M_{k-1}$. By N1, this implies $N \leq M$.

The proof of sufficiency of $(*)$ also consists of two steps:

S1. Reduce to the case when $\mathfrak{n} M=\mathfrak{n} N$, that is, when $R_{1}=S_{1}$. To do this we assume that $K \leq M$ and $\mathfrak{n} K \subset \mathfrak{n} M$, and we prove that there is $N \subset M$ such that $K \leq N$. In the most typical and most difficult case when $R_{2}-R_{1}>$ $-2 e$, we choose $N=M^{\prime}:=\{x \in M: x$ is not a norm generator $\}$. One can show that $M^{\prime}$ is a lattice with $\left[M: M^{\prime}\right]=\mathfrak{p}$.

S2. We then proceed with an induction on the dimension. Suppose that $N \leq$ $M$ but $N$ is not represented by $M$. We may assume that this pair of lattices is chosen so that ord volN - ord volM is minimal. By $\mathbf{S 1}$, we must have $\mathfrak{n} M=\mathfrak{n} N$, that is, $R_{1}=S_{1}$. In the most typical case we show that we can choose the BONGs of $M$ and $N$ such that $a_{1}=b_{1}$ and $\prec b_{2}, \ldots, b_{n} \succ \leq \prec a_{2}, \ldots, a_{n} \succ$. By the induction hypothesis this implies that $\prec b_{2}, \ldots, b_{n} \succ \rightarrow \prec a_{2}, \ldots, a_{n} \succ$. Together with $a_{1}=b_{1}$, this implies by [B. Lemma 2.2] that $N \rightarrow M$. 


\section{EFFECTIVENESS}

In order to apply Theorems 3.2 and 4.5, the first step we need to take is to write the two lattices in terms of some good BONGs. While arbitrary BONGs are easy to produce by simply using the definition of a BONG, for good BONGs we need to find some maximal norm splittings of the two lattices first. Then, by $\mathrm{B}$, Lemma 4.3(iii)], we can obtain a good BONG for a lattice by putting together the BONGs of the components in a maximal norm splitting. A method to produce maximal norm splitting is described in the proof of Lemmas 4.5 and 4.6 in $B$.

We now give an explicit algorithm for finding a maximal norm splitting of a lattice $L$. The first step is to take an arbitrary splitting $L=L_{1} \perp \ldots \perp L_{m}$ where each $L_{i}$ is either unary or binary modular and $\mathfrak{s} L_{1} \supseteq \ldots \supseteq \mathfrak{s} L_{m}$; see [M, $\left.\S 94\right]$ for details on how to obtain such a splitting. For the sake of convenience, we let $\operatorname{ord} \mathfrak{s} L_{i}=r_{i}$, ord $\mathfrak{n} L^{\mathfrak{s} L_{i}}=u_{i}$ and $\operatorname{ord} \mathfrak{n} L_{i}=v_{i}$. Then $L=L_{1} \perp \ldots \perp L_{m}$ is a maximal norm splitting if each component has maximal norm, i.e. $\mathfrak{n} L_{i}=\mathfrak{n} L^{\mathfrak{s} L_{i}}$ or, equivalently, $v_{i}=u_{i}$. Note that in general $\mathfrak{n} L_{i} \subseteq \mathfrak{n} L^{\mathfrak{s} L_{i}}$, i.e. $v_{i} \geq u_{i}$. The idea of the algorithm is to decrease at every step the number of indices $i$ for which $L_{i}$ does not have maximal norm, i.e. $v_{i}>u_{i}$. At each step we write the components $L_{i}$ that have maximal norms in terms of BONGs as $L_{i} \cong \prec a_{i} \succ$ or $L_{i} \cong \prec a_{i}, b_{i} \succ$ relative to some BONG $x_{i}$ or $x_{i}, y_{i}$, and all the other components in terms of matrices as $L_{i} \cong\left(\begin{array}{ll}a_{i} & b_{i} \\ b_{i} & c_{i}\end{array}\right)$ relative to some basis $x_{i}, y_{i}$. (Note that the unary components always have maximal norms.)

If $i \leq j$, then $L_{j}^{\mathfrak{s} L_{i}}=L_{j}$ and so ord $\mathfrak{n} L_{j}^{\mathfrak{s} L_{i}}=v_{j}$. If $i \geq j$, then $L_{j}^{\mathfrak{s} L_{i}}=\mathfrak{p}^{r_{i}-r_{j}} L_{j}$ and this implies ord $\mathfrak{n} L_{j}^{\mathfrak{s} L_{i}}=2\left(r_{i}-r_{j}\right)+v_{j}$. Therefore,

$$
u_{i}=\min \left(\left\{v_{j} \mid j \geq i\right\} \cup\left\{2\left(r_{i}-r_{j}\right)+v_{j} \mid j \leq i\right\}\right) .
$$

Take an index $i$ such that $v_{i}>u_{i}$. Then we have either $u_{i}=v_{j}$ for some $j>i$ or $u_{i}=2\left(r_{i}-r_{j}\right)+v_{j}$ for some $j<i$. In both cases one can show that $L_{j}$ has maximal norm, i.e. $v_{j}=u_{j}$. In fact we have a more precise result, namely, $v_{i}=\min \left\{v_{j}, 2\left(r_{i}-r_{\ell}\right)+v_{\ell}\right\}$, where $j$ is the smallest index $j>i$ such that $v_{j}=u_{j}$ and $\ell$ is the largest index $\ell<i$ such that $v_{\ell}=u_{\ell}$. (If $v_{j}>u_{j}$ for all $j>i$, then we ignore $v_{j}$; if $v_{\ell}>u_{\ell}$ for all $\ell<i$, then we ignore $2\left(r_{i}-r_{\ell}\right)+v_{\ell}$.)

We will show that the components $L_{i}$ and $L_{j}$ of the splitting can be replaced by $L_{i}^{\prime}$ and $L_{j}^{\prime}$ such that both $v_{i}=u_{i}$ and $v_{j}=u_{j}$ hold for the new splitting. Note that in this new splitting the number of components having maximal norm is increased by 1 . There are two cases to be considered.

Case 1. Suppose that $u_{i}=v_{j}$ for some $j>i$. We take $L_{i}^{\prime}=\mathcal{O}\left(x_{i}+x_{j}\right)+\mathcal{O} y_{i}$. Then $L_{i} \perp \mathcal{O} x_{j}=L_{i}^{\prime} \perp \mathcal{O} x_{j}^{\prime}$, and by comparing determinants we deduce that $Q\left(x_{j}^{\prime}\right)=a_{j}\left(\operatorname{det} L_{j}^{\prime}\right)^{-1} \operatorname{det} L_{j}$. Note that $L_{i} \perp L_{j}=L_{i}^{\prime} \perp L_{j}^{\prime}$, where $L_{j}^{\prime}=\prec x_{j}^{\prime} \succ$ if $L_{j}$ is unary, and $L_{j}^{\prime}=\prec x_{j}^{\prime}, y_{j} \succ$ if $L_{j}$ is binary. Hence $L_{j}^{\prime} \cong \prec a_{j}^{\prime} \succ$ or $\prec a_{j}^{\prime}, b_{j} \succ$, where $a_{j}^{\prime}=a_{j}\left(\operatorname{det} L_{j}^{\prime}\right)^{-1} \operatorname{det} L_{j}$. We have $\operatorname{det} L_{i}=a_{i} c_{i}-b_{i}^{2}$ and

$$
L_{i}^{\prime} \cong\left(\begin{array}{cc}
a_{i}+a_{j} & b_{i} \\
b_{i} & c_{i}
\end{array}\right)
$$

relative to the basis $x_{i}+x_{j}, y_{i}$ so $\operatorname{det} L_{i}^{\prime}=\left(a_{i}+a_{j}\right) c_{i}-b_{i}^{2}$. Also, $\mathfrak{n} L_{i}^{\prime}=\left(a_{i}+a_{j}\right) \mathcal{O}=$ $\mathfrak{p}^{u_{i}} ;$ so $L_{i}^{\prime} \cong \prec a_{i}+a_{j},\left(a_{i}+a_{j}\right)^{-1} \operatorname{det} L_{i}^{\prime} \succ$. (Note that $L_{i}^{\prime}$ has maximal norm; so it has to be written in terms of a BONG in our algorithm.) 
Case 2. Suppose that $u_{i}=2\left(r_{i}-r_{j}\right)+v_{j}$ for some $j<i$. For $1 \leq \ell \leq m$, let $u_{\ell}^{\sharp}=\operatorname{ord} \mathfrak{n}\left(L^{\sharp}\right)^{\mathfrak{s} L_{\ell}^{\sharp}}$ and $v_{\ell}^{\sharp}=\operatorname{ord} \mathfrak{n} L_{\ell}^{\sharp}$. We have $u_{i}=2\left(r_{j}-r_{i}\right)+v_{j}, v_{i}>u_{i}$ and $v_{j}=u_{j}$, and so

$$
v_{i}^{\sharp}=-2 r_{i}+v_{i}>-2 r_{i}+u_{i}=u_{i}^{\sharp} .
$$

By the same token, we have $v_{j}^{\sharp}=u_{j}^{\sharp}$. Furthermore,

$$
u_{i}^{\sharp}=-2 r_{i}+u_{i}=-2 r_{i}+2\left(r_{i}-r_{j}\right)+v_{j}=-2 r_{j}+v_{j}=v_{j}^{\sharp} .
$$

Thus the components $L_{i}^{\sharp}$ and $L_{j}^{\sharp}$ of the splitting $L^{\sharp}=L_{m}^{\sharp} \perp \ldots \perp L_{1}^{\sharp}$ are in the same situation as $L_{i}$ and $L_{j}$ were in Case 1 . Therefore, we can apply the procedure described in Case 1 to $L_{i}^{\sharp}=\mathfrak{p}^{-r_{i}} L_{i}$ and $L_{j}^{\sharp}=\mathfrak{p}^{-r_{j}} L_{j}$. As in Case 1, we have a splitting $L_{i}^{\sharp} \perp L_{j}^{\sharp}=K_{i} \perp K_{j}$ where $K_{i}$ and $K_{j}$ have the same scales and dimensions as $L_{i}^{\sharp}$ and $L_{j}^{\sharp}$, ord $\mathfrak{n} K_{i}=u_{i}^{\sharp}$ and $\operatorname{ord} \mathfrak{n} K_{j}=u_{j}^{\sharp}$. We then obtain $L_{i}^{\prime}$ and $L_{j}^{\prime}$ as $K_{i}^{\sharp}$ and $K_{j}^{\sharp}$, respectively. Now, $L_{i}^{\sharp}=\mathfrak{p}^{-r_{i}} L_{i} \cong \pi^{-2 r_{i}}\left(\begin{array}{c}a_{i} b_{i} \\ b_{i} c_{i}\end{array}\right)$ relative to the basis $\pi^{-r_{i}} x_{i}, \pi^{-r_{i}} y_{i}$, and $L_{j}^{\sharp} \cong \pi^{-2 r_{j}} \prec a_{j} \succ$ or $\pi^{-2 r_{j}} \prec a_{j}, b_{j} \succ$ relative to the BONG $\pi^{-r_{j}} x_{j}$ or $\pi^{-r_{j}} x_{j}, \pi^{-r_{j}} y_{j}$, respectively. By applying Case 1 and taking duals, one obtains $L_{i}^{\prime}=\mathcal{O}\left(x_{i}+\pi^{r_{i}-r_{j}} x_{j}\right)+\mathcal{O} y_{j}$ and $L_{j}^{\prime} \cong \prec a_{j}^{\prime} \succ$ or $\prec a_{j}^{\prime}, b_{j} \succ$ relative to some BONG $x_{j}^{\prime}$ or $x_{j}^{\prime}, y_{j}$, where $a_{j}^{\prime}=a_{j}\left(\operatorname{det} L_{i}^{\prime}\right)^{-1} \operatorname{det} L_{i}$. We have $\operatorname{det} L_{i}=a_{i} c_{i}-b_{i}^{2}$ and, since

$$
L_{i}^{\prime} \cong\left(\begin{array}{cc}
a_{i}+\pi^{2\left(r_{i}-r_{j}\right)} a_{j} & b_{i} \\
b_{i} & c_{i}
\end{array}\right)
$$

relative to the basis $x_{i}+\pi^{r_{i}-r_{j}} x_{j}, y_{i}$, $\operatorname{det} L_{i}^{\prime}=\left(a_{i}+\pi^{2\left(r_{i}-r_{j}\right)} a_{j}\right) c_{i}-b_{i}^{2}$ and, in terms of BONG, $L_{i}^{\prime} \cong \prec a_{i}+\pi^{2\left(r_{i}-r_{j}\right)} a_{j},\left(a_{i}+\pi^{2\left(r_{i}-r_{j}\right)} a_{j}\right)^{-1} \operatorname{det} L_{i}^{\prime} \succ$.

\section{RECOVERY OF RIEHM'S RESUlt}

The case when $M$ is unimodular considered by Riehm in $[\mathrm{R}]$ is somewhat simpler because, if we assume that condition (i) of Theorem 4.5 holds, then almost all indices are inessential. Therefore, conditions (ii) and (iii), which usually are the hardest to prove, need only be verified at a small number of indices. (See Lemmas 4.8 and [4.9.) Indeed, if $M$ is unimodular with $\mathfrak{n} M=\mathfrak{p}^{u}$, then the sequence $R_{1}, \ldots, R_{n}$ is $u,-u, u,-u, \ldots, u,-u$. It follows that for $1 \leq i \leq m-1$ we have $R_{i}+R_{i+1}=0$. Also note that $R_{i}=R_{i+2}$ for any $1 \leq i \leq n-2$. If condition (i) of Theorem 4.5 is satisfied, then $R_{3}=R_{1} \leq S_{1}$, which means that $i=2$ is not an essential index. Also if $3 \leq i \leq m-2$ and $i \leq n+1$, then by Lemma 4.6(i) we have $S_{i-2}+S_{i-1} \geq R_{i-2}+R_{i-1}=0=R_{i+1}+R_{i+2}$, and this implies that $i$ is not essential. Hence the only indices that could be essential are $i=1, m-1$ and $m$. Now $i=1$ and $m$ are always essential while $i=m-1$ is essential if and only if $R_{m}>S_{m-2}$. (Note that the index $m-1$ (resp. $m$ ) is ignored if $n<m-2$ (resp. $n<m-1)$.)

The most important result in $[\mathrm{R}]$ is the First MAin TheOREM, FMT for short. We now demonstrate the equivalence between conditions (1)-(3) of FMT and conditions (i)-(iv) of Theorem 4.5 .

Recall that in $[\mathbb{R}$ the two lattices under consideration are denoted by $L$ and $l$, and $L$ is unimodular. In FMT the two lattices have the same rank. The author used an additional lattice $l_{\mathfrak{m}}$. (For the definition of $L_{\mathfrak{a}}$ for some ideal $\mathfrak{a}$ see $[\mathbf{R}, 4.3]$.) The equivalence between $l \rightarrow L$ and $l_{\mathfrak{m}} \rightarrow L$ is obvious; it follows from the fact that 
$\mathfrak{m} \supseteq \mathfrak{n} L=\mathfrak{n} L^{\sharp}$. However, the necessary and sufficient conditions in terms of Jordan splittings are easier to state for $l_{\mathfrak{m}} \rightarrow L$.

Conditions (i) and (iv). These two conditions are in general easier to verify. If we assume that $R_{i}(L)=R_{i}$ and $R_{i}\left(l_{\mathfrak{m}}\right)=S_{i}$, then condition (i) implies $R_{1} \leq S_{1}$, $R_{n} \leq S_{n}$ and $R_{1}+R_{2} \leq S_{1}+S_{2}$; see also Lemma 4.6(i). We claim that altogether they are sufficient for condition (i). Indeed, condition (i) at $i=1$ and $n$ is just $R_{1} \leq S_{1}$ and $R_{n} \leq S_{n}$, respectively. If $2 \leq i \leq n-1$, we have $S_{i-1}+S_{i} \geq S_{1}+S_{2} \geq$ $R_{1}+R_{2}=0=R_{i}+R_{i+1}$ and (i) holds again. We claim that the three inequalities

$$
R_{1} \leq S_{1}, R_{n} \leq S_{n} \text { and } R_{1}+R_{2} \leq S_{1}+S_{2}
$$

are equivalent to $\mathfrak{s} L \supseteq \mathfrak{s} l_{\mathfrak{m}}, \mathfrak{n} L \supseteq \mathfrak{n} l_{\mathfrak{m}}$ and $\mathfrak{n} L^{\sharp} \subseteq \mathfrak{n} l_{\mathfrak{m}}{ }^{\sharp}$. We have ord $\mathfrak{n} L=R_{1}$ and ord $\mathfrak{n} L^{\sharp}=R_{1}\left(L^{\sharp}\right)=-R_{n}$. Analogous equalities hold for $l_{\mathfrak{m}}$. Therefore, $\mathfrak{n} L \supseteq$ $\mathfrak{n} l_{\mathfrak{m}}$ and $\mathfrak{n} L^{\sharp} \subseteq \mathfrak{n} l_{\mathfrak{m}}^{\sharp}$ are equivalent to $R_{1} \leq S_{1}$ and $R_{n} \leq S_{n}$. On the other hand, ord $\mathfrak{s} L=0=\left(R_{1}+R_{2}\right) / 2$ and, by [B, Corollary 4.4(iv)], ord $\mathfrak{s} l_{\mathfrak{m}}=\min \left\{S_{1}\right.$, $\left.\left(S_{1}+S_{2}\right) / 2\right\} ;$ so $\mathfrak{s} L \supseteq \mathfrak{s} l_{\mathfrak{m}}$ is equivalent to $\min \left\{S_{1},\left(S_{1}+S_{2}\right) / 2\right\} \geq\left(R_{1}+R_{2}\right) / 2$. But $R_{1}=u \geq 0=\left(R_{1}+R_{2}\right) / 2$; so $R_{1} \leq S_{1}$ implies $S_{1} \geq\left(R_{1}+R_{2}\right) / 2$. Therefore $\min \left\{S_{1},\left(S_{1}+S_{2}\right) / 2\right\} \geq\left(R_{1}+R_{2}\right) / 2$ if and only if $\left(S_{1}+S_{2}\right) / 2 \geq\left(R_{1}+R_{2}\right) / 2$ or $S_{1}+S_{2} \geq R_{1}+R_{2}$. The inclusions $\mathfrak{n} L \supseteq \mathfrak{n} l_{\mathfrak{m}}$ and $\mathfrak{n} L=\mathfrak{n} L^{\sharp} \subseteq \mathfrak{n} l_{\mathfrak{m}}{ }^{\sharp}$ follow from $\mathfrak{g} l_{\mathfrak{m}} \subseteq \mathfrak{g} L \subseteq \mathfrak{g} l_{\mathfrak{m}}^{\sharp}$, which is a part of FMT (1). Now $\mathfrak{s} L \supseteq \mathfrak{s} l_{\mathfrak{m}}$ is not a part of FMT (1) but $\mathfrak{s} L \supseteq \mathfrak{s} l$ is. But $l \subseteq l_{\mathfrak{m}}$; therefore $\mathfrak{s} L \supseteq \mathfrak{s} l_{\mathfrak{m}}$ implies $\mathfrak{s} L \supseteq \mathfrak{s} l$. Conversely, one can show that $\mathfrak{s} L \supseteq \mathfrak{s} l_{\mathfrak{m}}$ follows from $\mathfrak{s} L \supseteq \mathfrak{s} l$ and $\mathfrak{n} l_{\mathfrak{m}} \subseteq \mathfrak{n} L \subseteq \mathfrak{n} l_{\mathfrak{m}}{ }^{\sharp}$. (In fact, $\mathfrak{s} l_{\mathfrak{m}}=\mathfrak{s} l$ in most cases.)

Condition (iv) is vacuous in this case because if $S_{i} \geq R_{i+2}>S_{i-1}+2 e \geq$ $R_{i+1}+2 e$, then $R_{i+1}-R_{i}=R_{i+1}-R_{i+2}<-2 e$, which is impossible.

Conditions (ii) and (iii). Recall that the only essential indices for $L$ and $l_{\mathfrak{m}}$ are $1, n$ and, possibly, $n-1$. By Lemma 4.8, condition (ii) needs only be verified at $i=1, n-1$ and, if the index $n-1$ is essential, at $i=n-2$. By Lemma 4.9, condition (iii) only needs to be verified at $i=n-1$ and only if $n-1$ is essential. We now state the equivalent conditions from FMT, assuming that (i) holds. However, the proofs are too lengthy to include here.

Condition (ii) at $i=1$ and $n-1$ is equivalent to $\mathfrak{g} l_{\mathfrak{m}} \subseteq \mathfrak{g} L$ and $\mathfrak{g} L \subseteq \mathfrak{g} l_{\mathfrak{m}}{ }^{\sharp}$, respectively. If $R_{n} \leq S_{n-2}$, that is, if $n-1$ is not an essential index, then no additional conditions are required. One can prove that $R_{n} \leq S_{n-2}$ whenever $\mathfrak{m}=$ $\mathfrak{n} l^{\mathfrak{s}}$ or $\mathfrak{s}=\mathfrak{s} l_{\mathfrak{m}}^{\sharp}$. So condition (ii) at $i=n-2$ and condition (iii) at $i=n-1$ only have to be verified if $\mathfrak{n} l^{\mathfrak{s}} \subset \mathfrak{m}$ and $\mathfrak{s} \subset \mathfrak{s} l_{\mathfrak{m}}^{\sharp}$.

In the case when $\mathfrak{n} l^{\mathfrak{s}} \subset \mathfrak{m}$ and $\mathfrak{s}=\mathfrak{s} l_{\mathfrak{m}}^{\sharp}$, we have $l_{\mathfrak{m}}^{\sharp \mathfrak{s}}=l_{\mathfrak{m}}^{\sharp}$ and the condition $\mathfrak{g} L \subseteq \mathfrak{g}\langle a\rangle+\mathfrak{g} l_{\mathfrak{m}}{ }^{\sharp \mathfrak{s}}$ from FMT (2) is superfluous, since it follows from $\mathfrak{g} L \subseteq \mathfrak{g} l_{\mathfrak{m}}{ }^{\#}$. If $\mathfrak{n} l^{\mathfrak{s}} \subset \mathfrak{m}$ and $\mathfrak{s} \subset \mathfrak{s l}_{\mathfrak{m}}{ }^{\sharp}$, then one can show that parts (2) and (3) of FMT are equivalent to condition (ii) at index $n-2$ and condition (iii) at index $n-1$, respectively.

Acknowledgements. I want to thank Wai Kiu Chan for his interest in the subject and for pointing out the most important results that should be included in this announcement. His many comments and suggestions greatly improved the quality of the exposition. 


\section{REFERENCES}

[B] C. N. Beli, Integral spinor norms over dyadic local fields, J. Number Theory 102 (2003), 125-182. MR1994477 (2004i:11030)

[B1] C. N. Beli, BONG version of O'Meara's Theorem 93:28, preprint.

[B2] C. N. Beli, Representations of quadratic lattices over dyadic local fields, preprint.

$[\mathrm{OM}]$ O. T. O'Meara, Introduction to quadratic forms, Springer-Verlag, Berlin, 1963. MR 0152507 (27:2485)

[OM1] O. T. O'Meara, The integral representation of quadratic forms over local fields, Amer. J. Math. 80 (1958), 843-878. MR0098064 (20:4526)

[R] C. Riehm, On the integral representations of quadratic forms over local fields, Amer. J. Math. 86 (1964), 25-62. MR0161853 (28:5057)

Institute of Mathematics "Simion Stollow" of the Romanian Academy, 21 Calea Grivitei Street, 010702-Bucharest, Sector 1, Romania

E-mail address: raspopitu1@yahoo.com 